Gefässchirurgie 2020 $25: 654-659$ https://doi.org/10.1007/s00772-020-00689-2 Angenommen: 16. August 2020 Online publiziert: 18 . September 2020 ๑) Der/die Autor(en) 2020

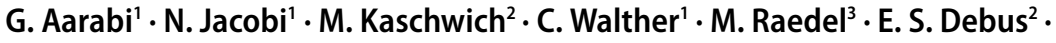
A. Larena-Avellaneda ${ }^{2} \cdot$ U. Seedorf ${ }^{1} \cdot$ G. Heydecke ${ }^{1} \cdot$ C.-A. Behrendt ${ }^{2}$ ' Poliklinik für Zahnärztliche Prothetik, Zentrum für Zahn-, Mund- und Kieferheilkunde, Universitätsklinikum Hamburg-Eppendorf, Hamburg, Deutschland

${ }^{2}$ Forschungsgruppe GermanVasc, Klinik und Poliklinik für Gefäßmedizin, Universitäres Herz- und Gefäßzentrum UKE Hamburg, Universitätsklinikum Hamburg-Eppendorf, Hamburg, Deutschland ${ }^{3}$ Abteilung für Zahnärztliche Prothetik, Medizinische Fakultät Carl Gustav Carus, Technische Universität Dresden, Dresden, Deutschland

\title{
Besteht ein Zusammenhang zwischen der peripheren arteriellen Verschlusskrankheit und Parodontitis?
}

\section{Chancen und Limitationen für eine interdisziplinäre Zusammenarbeit von Gefäßmedizin und Zahnmedizin}

nen, Entzündungen, gehemmte Angiogenese und reduzierte Mikrozirkulation zur IC und CLTI führen $[24,38]$.

Die krankheitsbezogene Lebensqualität ist aufgrund eingeschränkter körperlicher Funktionen (z.B. Mobilitätseinschränkungen, Körperpflege) und Schmerzen reduziert. Auch die emotionale Rollenfunktion, die soziale Funktionsfähigkeit und das psychische Wohlbefinden sind stark eingeschränkt [42, 54]. Patienten mit einer PAVK haben ein deutlich erhöhtes Sterblichkeitsrisiko, was nicht zuletzt aufgrund der deutlichen Assoziation mit koronaren und zerebrovaskulären Gefäßerkrankungen zusammenhängt [11]. Mindestens $30 \%$ der PAVK-Patienten leiden an einer linksventrikulären Herzinsuffizienz [53]. Insgesamt haben PAVK-Patienten ein etwa dreifach erhöhtes Risiko, an einer kardiovaskulären Komplikation zu sterben [16]. Weitere klinisch relevante Begleiterkrankungen sind chronische Niereninsuffizienz bis zur Dialysepflichtigkeit und ein Diabetes mellitus [34].

Risikofaktoren wie Rauchen, Hypertonie, Diabetes mellitus, Dyslipidämie sowie chronisch entzündliche Erkran- kungen wie Parodontitis (PA) erhöhen das Risiko an einer PAVK zu erkranken und haben einen Einfluss auf den Krankheitsverlauf [28, 55].

Die schwere Form der PA war im Jahr 2010 weltweit die sechsthäufigste Erkrankung und betraf 743 Mio. Menschen [29]. Durch eine Dysbiose der Mikroorganismen im subgingivalen Biofilm entsteht eine Entzündung des Hart- und Weichgewebes um den Zahn [21]. Die starke Biofilmakkumulation bewirkt eine übermäßige Entzündungsreaktion und Immunantwort des Wirts [51]. Die Epithelzellen stellen normalerweise eine physikalische Barriere dar. Einige parodontopathogene Bakterien durchdringen diese Barriere: Porphyromonas gingivalis synthetisiert verschiedene Virulenzfaktoren, die durch proteolytische Inaktivierung von Schlüsselkomponenten das Wirtsabwehrsystem umgehen [13]. Anschließend durchdringen die Mikroorganismen die vaskulären Läsionen und vermehren sich [45]. Dies führt zu einer chronisch erhöhten Produktion und Freisetzung proinflammatorischer Mediatoren (z. B. IL-6, IL-1 $\beta$, TNF $\alpha$ ), C-reaktivem Protein (CRP) und Bildung von 


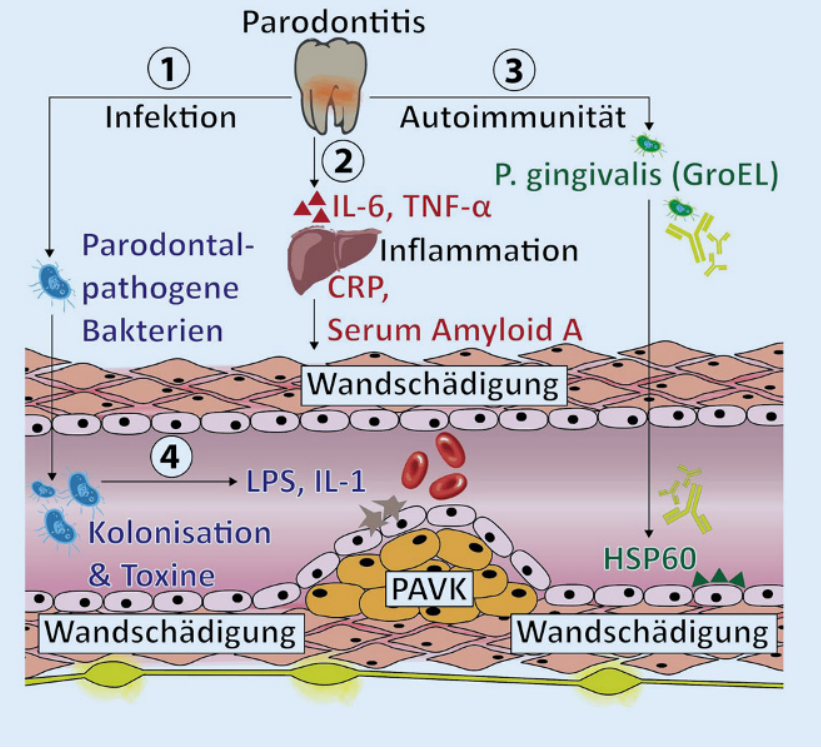

Abb. $1<$ Schematische (unvollständige) Darstellung der pathogenen Mechanismen und Assoziation zwischen Parodontitis und peripherer arterieller Verschlusskrankheit. IL-6/IL-1 Interleukin-6, Interleukin-1, LPS Lipopolysaccharid, TNF-a Tumornekrosefaktor-Alpha, CRPC-reaktives Protein, HSP60 Hitzeschockprotein-60, PAVK periphere arterielle Verschlusskrankheit
Fibrinogen in der Leber $[1,2,48]$. Die zunächst lokal begrenzten Entzündungsprozesse können sich systemisch ausbreiten und Entzündungen an Gefäßwänden auslösen $[8,40]$. In mehreren Studien wurden bereits parodontopathogene Bakterien in arteriosklerotischen Läsionen gefunden [15, 32, 44].

Interessant in diesem Zusammenhang ist, dass sich die Risikofaktoren der PA und der PAVK teilweise überschneiden. Beide Erkrankungen werden durch allgemein akzeptierte kardiovaskuläre Risikofaktoren, wie zum Beispiel Rauchen und Diabetes mellitus, beeinflusst [52]. Zudem wurden bisher verschiedene molekulare Marker dokumentiert: Proatherogene Lipidprofile [19] sowie systemisch erhöhte CRP-Werte [52] sind nachweislich bei beiden Erkrankungen als Zeichen chronischer Entzündungsprozesse erhöht.

\section{Aktuelle Daten zur Pathophysiolo- gie}

In der bestehenden Literatur wurden vier pathogene Mechanismen zur Entstehung einer PAVK im Zusammenhang mit einer PA erarbeitet (• Abb. 1; [2, 28, 30]):

1. Parodontale Bakterien können sich über den Blutstrom oder über die Lymphbahnen im Gefäßsystem ausbreiten und die Arterienwand schädigen [2, 7, 30, 47], wodurch es zu einer Initiation der Atherosklerose kommt.

2. Entzündungsmediatoren werden von einer Entzündung im Mund in den Blutstrom freigesetzt. Interleukin-6 und TNFa gelangen durch den Blutstrom in die Leber und induzieren eine vermehrte Produktion von CRP und Fibrinogen, wodurch eine Akut-Phase-Reaktion ausgelöst wird, die proinflammatorisch sowie proatherogen wirkt [48].

3. Spezifische bakterielle Antigene können eine Autoimmunität auslösen, die gegen das Hitzeschockprotein 60 (HSP60) gerichtet ist. Es konnte gezeigt werden, dass PA-Patienten vermehrt Antikörper und T-Zellen gegen HSP60 bilden [35], das sich auf der Oberfläche von Endothelund vaskulären glatten Muskelzellen befindet. Die gegen HSP60-gerichtete Autoimmunantwort löst die endotheliale Dysfunktion aus und verstärkt die chronische Entzündung in der Arterienwand [2, 22, 47].

4. Orale pathogene Bakterien produzieren Endotoxine, wie Lipopolysaccharide (LPS), die einen proatherogenen und proinflammatorischen Effekt haben [46]. Durch LPS werden Entzündungsmediatoren wie IL-1 produziert. Dies führt zu einer vermehrten Fibrinakkumulation, Reduktion der Fibrinolyse, Anheftung von Blutplättchen und Akkumulation von Gewebsmakrophagen im Blutgefäß. Makrophagen wandeln sich zu Schaumzellen um und vergrößern so die atherosklerotische Plaque [31].

Bisher gibt es in der Literatur keinen validen Nachweis für einen kausalen Zusammenhang beider Erkrankungen. Es liegen allerdings Hinweise vor, dass die Parodontitis einer Erstdiagnose von PAVK vorausgeht.

\section{Evidenzbasis zur Assoziation beider Erkrankungen}

Die Studienlage zur Assoziation von PA mit der PAVKzeigt, dass es eine Reihe von Publikationen über den Zusammenhang zwischen PA bzw. oralen Erkrankungen und Atherosklerose im Allgemeinen gibt. Hierunter beleuchten nur wenige einen Zusammenhang zwischen PA und PAVK im Speziellen.

Das Design dieser wenigen Untersuchungen ist sehr heterogen und reicht von kleinen Fallkontrollstudien mit nur 57 Teilnehmern [17] bis zu Kohortenstudien mit über 500.000 Teilnehmern [18].

Als Maß für die Stärke der Assoziation zwischen PA und PAVK wurden in den Analysen meist durch logistische Regressionsanalysen bestimmte standardisierte Effektgrößen, wie Odds Ratio (OR), Hazard Ratio (HR) bzw. Relatives Risiko (RR) zusammen mit einem 95\%-Konfidenzintervall (CI) als Vertrauensmaß beschrieben. Nach Anpassung an Alter, Geschlecht und wichtige kardiovaskuläre Risikofaktoren reicht die Bandbreite der berichteten ORs von 2,03 in der Querschnittsstudie von Y-B Ahn et al. [6] bis zu mehr als 8,18 in der Fallkontrollstudie von U Soto-Barreras et al. [50]. Im Allgemeinen ergaben die kleineren Fall-Kontroll-Studien höhere ORs als die größeren Kohortenstudien. Berechnungen des HR oder des RR sind nur aus wenigen Publikationen verfügbar. Die derzeit aktuellste wurde von D-H Cho et al. [18] durchgeführt. Hierbei handelt es sich um eine retrospektive gematchte Kohortenstudie, in der 514.832 Koreaner zwischen 40 und 79 Jahren eingeschlossen wurden. Die Autoren geben einen HR von 
1,15 für die Entwicklung einer PAVK in der PA-Gruppe verglichen mit der entsprechenden Kontrollgruppe an. Daten von FJ Muñoz-Torres et al. [43] aus der Nurses' Health Study zeigten einen signifikanten Zusammenhang zwischen Zahnverlust und PAVK und berichteten über eine $\mathrm{HR}$ von 1,3 für PAVK bei Frauen mit PA im Vergleich zu Frauen ohne PA (Kontrollgruppe). Allerdings wurde keine Anpassung bezüglich des Rauchens als gemeinsamen Risikofaktor vorgenommen. Die Assoziation beider Erkrankungen mit Rauchen ist allerdings hinreichend bekannt $[5,36]$. Des Weiteren wurden ausschließlich Teilnehmer eines Geschlechts eingeschlossen, was ebenfalls die Aussagekraft dieser Studie limitiert. Gleiches gilt für die Studien von H-C Hung et al. [25], DG Bloemenkamp et al. [12] und MV Mendez et al. [39]. Eine weitere Herausforderung liegt in der komplexen, chronisch progressiven Natur der PAVK. Es gibt zahlreiche Hinweise darauf, dass substanzielle Unterschiede zwischen den Risikoprofilen und Langzeitergebnissen von Patienten mit IC vs. CLTI existieren. Gleichermaßen bestehen Hinweise auf Unterschiede zwischen beiden Geschlechtern. Dementsprechend ist es mitunter erforderlich, die Analysen zu stratifizieren oder die Auswertungen zu adjustieren. Die von S Yang et al. [55] veröffentlichte Metaanalyse berichtete von einem statistisch signifikanten RR von 1,7 für die Entwicklung einer PAVK bei Patienten mit PA im Vergleich zu Patienten ohne PA.

Zusammenfassend deuten die Ergebnisse aktueller und älterer Studien darauf hin, dass eine PA das Risiko für die Entwicklung einer PAVK erhöht. • Tab. 1 gibt eine Übersicht der aktuellen Studienlage.

\section{Parodontalbehandlung und Schweregrad der PAVK}

Aktuell gibt es kaum Analysen auf der Grundlage von Routinedaten, die sich mit der Assoziation zwischen chronischen oralen Entzündungen und der PAVK beschäftigt haben. Außerdem haben nur wenige Studien untersucht, $\mathrm{ob}$ es eine wechselseitige Assoziation zwischen der Behandlung der PA und dem Schweregrad der PAVK gibt. In

Gefässchirurgie 2020 - 25:654-659 https://doi.org/10.1007/s00772-020-00689-2

(c) Der/die Autor(en) 2020

G. Aarabi - N. Jacobi - M. Kaschwich · C. Walther - M. Raedel · E. S. Debus · A. LarenaAvellaneda $\cdot$ U. Seedorf · G. Heydecke · C.-A. Behrendt

Besteht ein Zusammenhang zwischen der peripheren arteriellen Verschlusskrankheit und Parodontitis? Chancen und Limitationen für eine interdisziplinäre Zusammenarbeit von Gefäßmedizin und Zahnmedizin

\section{Zusammenfassung}

Die periphere arterielle Verschlusskrankheit (PAVK) ist die häufigste Durchblutungsstörung peripherer Gefäße. Die PAVK wird durch Risikofaktoren und Komorbiditäten, wie zum Beispiel Rauchen, Bluthochdruck, Dyslipidämie, Diabetes und chronische Niereninsuffizienz begünstigt. Epidemiologische Studien weisen allerdings auch darauf hin, dass zusätzlich die Parodontitis (PA) das Risiko für eine PAVK erhöhen kann. Die PA ist eine häufige, durch bakteriellen Biofilm ausgelöste, chronische Entzündung des Zahnhalteapparates. Parodontitis wird durch mangelhafte Mundhygiene, Rauchen und Diabetes begünstigt und die Prävalenz steigt mit dem Alter an. Beide Erkrankungen bzw. deren Symptome sind therapierbar, wenn die Behandlung frühzeitig einsetzt. Deshalb ist die Früherkennung besonders wichtig.

Is there a connection between peripheral arterial occlusive disease and periodontitis? Opportunities for and limitations of an interdisciplinary collaboration between vascular medicine and dentistry

\section{Abstract}

Peripheral arterial occlusive disease (PAOD) is the most common disorder of arterial blood flow to the peripheral arteries. PAOD is promoted by risk factors and comorbidities, such as smoking, high blood pressure, dyslipidemia, diabetes, and chronic renal failure. Epidemiological studies indicate that periodontitis can increase the risk of PAOD. Periodontitis is a common, chronic inflammation of the periodontium caused by accumulation of bacterial biofilm. It is promoted by poor oral hygiene, smoking, and diabetes, and the prevalence increases with age. Both diseases and/or their symptoms can be treated if therapy starts early. Thus, early detection is particularly important. Although
Obwohl der Nachweis einer kausalen Rolle der PA für die Entstehung der PAVK noch aussteht, unterstützen die in diesem Artikel zusammengefassten Daten den Vorschlag einer interdisziplinären Zusammenarbeit zwischen Gefäß- und Zahnmedizinern. Es kann in der gefäßmedizinischen Praxis erwogen werden, PAVK-Patienten, unabhängig vom Stadium ihrer Erkrankung, zwecks Diagnose und Behandlung einer PA an Zahnärzt*innen zu verweisen. Umgekehrt sollten auch Zahnärzt*innen erwägen, Patienten, die an einer schweren Form der PA leiden, an Gefäßmediziner zu verweisen.

Schlüsselwörter

Versorgungsforschung $\cdot$ Prävention · Periphere arterielle Verschlusskrankheit · Parodontitis einer kürzlich veröffentlichten Studie wurden Routinedaten der Sozialversicherungsträger (BARMER) analysiert, um herauszufinden, ob eine PA-Behandlung den Schweregrad einer PAVK beeinflussen kann [3]. Dabei wurde there is still no evidence of a causal role of periodontitis in the development of PAOD, the data summarized in the current article support the suggestion of a professional collaboration between physicians in vascular care and dentists. Referring PAOD patients to the dentist for diagnosis and treatment of periodontitis should be considered, regardless of the stage of their disease. Conversely, dentists should consider referring patients suffering from a severe form of periodontitis to vascular specialists.

\section{Keywords}

Health care research · Prevention · Peripheral arterial occlusive disease $\cdot$ Periodontitis 


\begin{tabular}{|c|c|c|c|c|}
\hline Autor & $\begin{array}{l}\text { Studien- } \\
\text { design }\end{array}$ & $\begin{array}{l}\text { Stärke der Assoziation zwischen PA bzw. } \\
\text { Zahnverlust und PAVK }\end{array}$ & $\begin{array}{l}\text { Eingeschlossene } \\
\text { Teilnehmer }\end{array}$ & Limitationen \\
\hline D-HCho et al. [18] & KS & $H R=1,15(95 \% \mathrm{Cl}: 1,0-1,2)$ & 514.832 & - \\
\hline Yang et al. [55] & SR, M & $\begin{array}{l}R R=1,70(95 \% \mathrm{Cl}: 1,3-2,3 ; p=0,01)^{\mathrm{a}} \mathrm{WMD}=3,75 \\
(95 \% \mathrm{Cl}: 1,3-6,2 ; p=0,003)\end{array}$ & 4307 & - \\
\hline MU Calapkorur et al. [14] & QS & $O R=5,8(95 \% \mathrm{Cl}: 1,5-21,9 ; p=0,009)$ & 60 & - \\
\hline FJ Muñoz-Torres et al. [43] & KS & $H R=1,3(95 \% \mathrm{Cl}: 1,0-1,7)$ & 79.663 & $\begin{array}{l}\text { Keine Anpassung für Rauchen, } \\
\text { nur Frauen eingeschlossen }\end{array}$ \\
\hline Y-B Ahn et al. [6] & QS & $O R=2,0(95 \% \mathrm{Cl}: 1,0-3,9 ; p=0,036)$ & 1343 & - \\
\hline U Soto-Barreras et al. [50] & FS & $O R=8,2(95 \% \mathrm{Cl}: 1,2-35,2 ; p=0,031)$ & 60 & - \\
\hline Y-W Chen et al. [17] & FS & $O R=5,5(95 \% \mathrm{Cl}: 1,6-18,9 ; p=0,007)$ & 57 & - \\
\hline B Lu et al. [37] & QS & $O R=2,3(95 \% \mathrm{Cl}: 1,2-4,2 ; p=0,004)$ & 3585 & - \\
\hline J Molloy et al. [41] & FS & $\begin{array}{l}{ }^{\mathrm{b}} \text { Vascular disease } p \text {-value } 0,014 \\
{ }^{\mathrm{b}} \text { Vascular surgery } p \text {-value } 0,001\end{array}$ & 2006 & - \\
\hline H-C Hung et al. [25] & KS & $R R=1,41(95 \% \mathrm{Cl}: 1,1-1,8)$ & 45.136 & Nur Männer eingeschlossen \\
\hline DG Bloemenkamp et al. [12] & FS & $O R=3,0(95 \% \mathrm{Cl}: 1,4-6,3)$ & 687 & Nur Frauen eingeschlossen \\
\hline MV Mendez et al. [39] & KS & $O R=2,27(95 \% \mathrm{Cl}: 1,3-3,9 ; p=0,003)$ & 1110 & Nur Männer eingeschlossen \\
\hline \multicolumn{5}{|c|}{$\begin{array}{l}\text { PAVK periphere arterielle Verschlusskrankheit, PA Parodontitis, RR Risk Ratio, OR Odds Ratio, HR Hazard Ratio, WMD „weighted mean difference“, KS Kohor- } \\
\text { tenstudie, } S R \text { systematisches Review, } M \text { Metaanalyse, QS Querschnittsstudie, FS Fallkontrollstudie, CI Konfidenzintervall } \\
\text { aPAVK-Patienten hatten einen vermehrten Zahnverlust im Vergleich zu Patienten ohne PAVK } \\
\text { 'Bedingungen, die signifikant mit Zahnverlust assoziiert waren }\end{array}$} \\
\hline
\end{tabular}

rungen für die nicht chirurgische und die chirurgische Behandlung der PA bewertet. Bei der nicht chirurgischen Intervention erhielten die Patienten ein konventionelles subgingivales Debridement (mechanische Entfernung des subgingivalen Biofilms mit anschließender Wurzelglättung). Die zahnchirurgische resektive Intervention der PA umfasste das Säubern der tiefen Taschen unter Sicht. Die Studie ergab, dass die hospitalisierten PAVK-Patienten, die eine PABehandlung erhielten, einen deutlich geringeren Anteil an der klinisch fortgeschrittenen CLTI-Form (28,76\% unter den Behandelten gegenüber 52,12\% unter den Nichtbehandelten) aufwiesen als diejenigen, bei denen keine PA-Behandlung durchgeführt wurde. Multivariable Regressionsanalysen ergaben, dass die Chance für das Vorhandensein der CLTI (vs. IC) nach Adjustierung für Alter, Geschlecht und Diabetes bei den nicht PA-behandelten Patienten 1,97 (95\% CI 1,83-2,13) betrug. Dieses Ergebnis weist darauf hin, dass die Behandlung der PA bei den hospitalisierten PAVK-Patienten mit früheren PAVK-Stadien assoziiert ist, unabhängig von Alter, Geschlecht und Diabetes. Um mögliche kausale Effekte der Behandlung der PA auf den Verlauf der PAVK weiter abzuklären, wären aber
Daten aus randomisierten kontrollierten Interventionsstudien wünschenswert, die aktuell jedoch noch nicht vorliegen.

\section{Diskussion}

Patienten mit einer PAVK haben neben einer schlechteren krankheitsspezifischen Lebensqualität ein deutlich erhöhtes Risiko schwerer kardiovaskulärer Ereignisse. Vorrangiges Ziel der Früherkennung ist die Einleitung einer optimalen leitliniengerechten Therapie mit konsequenter Optimierung der Risikofaktoren und adäquater Arzneimittelversorgung.

Eine zentrale Rolle in der Krankheitsentstehung und Progression stellen Komorbiditäten, wie Diabetes (ca. 30\%) und Niereninsuffizienz (ca. 25\%) dar, deren chronische Entzündung als mitursächlich diskutiert werden. Die in diesem Beitrag dargestellten Ergebnisse weisen außerdem darauf hin, dass chronische orale Entzündungen, insbesondere die PA, ebenfalls eine Rolle spielen könnten. Die PA ist eine der häufigsten Entzündungserkrankungen des Menschen betroffen von einer schweren Form der Erkrankung sind ca. 10-20\% der über 40-Jährigen [27]. Die Diagnose erfolgt häufig erst spät, da die Erkrankung lan- ge symptomlos bleibt. Insbesondere $\mathrm{Pa}$ tienten mit Vorerkrankungen profitieren von einer frühzeitigen Diagnostik und konsequenten Therapie, da die chronische Entzündung das Risiko steigert, an Schlaganfall, Herzinfarkt und Arthritis zu erkranken. Das Risiko für Frühgeburten steigt bei werdenden Müttern, die an einer schweren Form der PA leiden, um das Siebenfache [26].

Vor dem Hintergrund aller Indizien kann es sinnvoll sein, die interdisziplinäre Zusammenarbeit zwischen Gefäßmedizinern und Zahnmedizinern in Deutschland systematisch $\mathrm{zu}$ verbessern. Dafür wäre es sinnvoll, die rechtlichen Voraussetzungen dafür zu schaffen, dass sich diese beiden Fachgruppen in Deutschland einander Patienten überweisen können. Eine manifeste PA kann im Rahmen einer zahnmedizinischen Untersuchung schnell und sicher diagnostiziert und, falls nötig, nachfolgend therapiert werden. Aktuell fehlt es aber noch an Studienergebnissen, beispielsweise aus randomisierten kontrollierten Interventionsstudien, die den therapeutischen Erfolg auf die Entstehung und den Verlauf der PAVK nachweisen.

Die Mundgesundheit spielt in den aktuellen Leitlinien zur Diagnostik und Behandlung der PAVK bisher kaum eine 
Rolle [4, 23]. Die in diesem Artikel dargestellten Ergebnisse bezüglich eines möglichen Zusammenhangs zwischen chronischen oralen Entzündungen, insbesondere der PA, und der PAVK lassen es aber sinnvoll erscheinen, eine wechselseitige fachärztliche bzw. zahnärztliche Vorstellung zu erwägen. Dies gilt besonders für die über 65-Jährigen sowie Patienten mit ausgeprägtem Risikoprofil wie Rauchen und Diabetes, die sowohl die PAVK als auch die PA negativ beeinflussen. Diese Erwägung erscheint sinnvoll, obwohl der Nachweis eines kausalen Zusammenhangs zwischen der PA und der PAVK noch aussteht.

\section{Fazit für die Praxis}

- Sowohl die PAVK und vaskuläre Komplikationen des Diabetes als auch die PA sind zahlenmäßig ein relevantes globales Gesundheitsproblem mit Beeinträchtigung der Lebensqualität und Allgemeingesundheit der betroffenen Patienten.

- Das Risiko- und Komorbiditätsprofil von Patienten, die an einer PA oder PAVK erkrankt sind, weist zahlreiche Überschneidungen (z. B. Diabetes, Rauchen) auf.

- Die in diesem Artikel dargestellten Ergebnisse unterstützen einen $\mathrm{Zu}$ sammenhang zwischen chronischen oralen Entzündungen, insbesondere der PA, und der PAVK.

- Studienergebnisse, beispielsweise aus randomisierten kontrollierten Interventionsstudien, die den therapeutischen Erfolg der PA-Behandlung auf die Entstehung und den Verlauf der PAVK nachweisen, stehen aktuell noch aus.

- Dennoch erscheint die Empfehlung, PAVK-Patienten unabhängig vom Stadium ihrer Erkrankung, zwecks Diagnose und Behandlung einer etwaig vorliegenden PA an den Zahnarzt zu verweisen, sinnvoll.

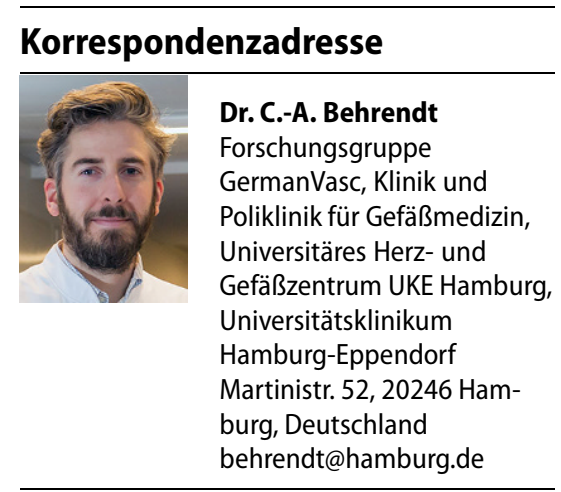

Funding. Open Access funding provided by Projekt DEAL.

\section{Einhaltung ethischer Richtlinien}

Interessenkonflikt. G. Aarabi, N. Jacobi, M. Kaschwich, C. Walther, M. Raedel, E.S. Debus, A. Larena-Avellaneda, U. Seedorf, G. Heydecke und C.A. Behrendt geben an, dass kein Interessenkonflikt besteht.

Für diesen Beitrag wurden von den Autoren keine Studien an Menschen oder Tieren durchgeführt. Für die aufgeführten Studien gelten die jeweils dort angegebenen ethischen Richtlinien.

Open Access. Dieser Artikel wird unter der Creative Commons Namensnennung 4.0 International Lizenz veröffentlicht, welche die Nutzung, Vervielfältigung, Bearbeitung, Verbreitung und Wiedergabe in jeglichem Medium und Format erlaubt, sofern Sie den/die ursprünglichen Autor(en) und die Quelle ordnungsgemäß nennen, einen Link zur Creative Commons Lizenz beifügen und angeben, ob Änderungen vorgenommen wurden.

Die in diesem Artikel enthaltenen Bilder und sonstiges Drittmaterial unterliegen ebenfalls der genannten Creative Commons Lizenz, sofern sich aus der Abbildungslegende nichts anderes ergibt. Sofern das betreffende Material nicht unter der genannten Creative Commons Lizenz steht und die betreffende Handlung nicht nach gesetzlichen Vorschriften erlaubt ist, ist für die oben aufgeführten Weiterverwendungen des Materials die Einwilligung des jeweiligen Rechteinhabers einzuholen.

Weitere Details zur Lizenz entnehmen Sie bitte der Lizenzinformation auf http://creativecommons.org/ licenses/by/4.0/deed.de.

\section{Literatur}

1. Aarabi G, Eberhard J, Reissmann DR et al (2015) Interaction between periodontal disease and atherosclerotic vascular disease-fact or fiction? Atherosclerosis 241:555-560

2. Aarabi G, Heydecke G, Seedorf U (2018) Roles of oral infections in the pathomechanism of atherosclerosis. Int J Mol Sci 19:1978

3. Aarabi G, Raedel M, Kreutzburg T et al (2020) Periodontal treatment and peripheral arterial disease severity - a retrospective analysis of health insurance claims data. Vasa 49:128-132

4. Aboyans V, Ricco JB, Bartelink MEL et al (2018) Editor's choice-2017 ESC guidelines on the diagnosis and treatment of peripheral arterial diseases, in collaboration with the European society for vascular surgery (ESVS). Eur J Vasc Endovasc Surg 55(3):305-368. https://doi.org/10. 1016/j.ejvs.2017.07.018

5. Agarwal S (2009) The association of active and passive smoking with peripheral arterial disease: results from NHANES 1999-2004. Angiology 60:335-345

6. Ahn Y-B, Shin M-S, Han D-H et al (2016) Periodontitis is associated with the risk of subclinical atherosclerosis and peripheral arterial disease in Korean adults. Atherosclerosis 251:311-318

7. Armingohar Z, Jørgensen JJ, Kristoffersen AK et al (2014) Bacteria and bacterial DNA in atherosclerotic plaque and aneurysmal wall biopsies from patients with and without periodontitis. J Oral Microbiol 6:23408

8. Armitage GC (2004) Periodontal diagnoses and classification of periodontal diseases. Periodontology 34:9-21

9. Behrendt CA, Bischoff MS, Schwaneberg T et al (2019) Population based analysis of gender disparities in 23,715 percutaneous endovascular revascularisations in the Metropolitan area of Hamburg. Eur JVasc Endovasc Surg 57:658-665

10. Behrendt CA, Sigvant B, Szeberin Z et al (2018) International variations in amputation practice: a VASCUNET report. Eur J Vasc Endovasc Surg 56:391-399

11. Bhatt DL, Steg PG, Ohman EM et al (2006) International prevalence, recognition, and treatment of cardiovascular risk factors in outpatients with atherothrombosis. JAMA 295:180-189

12. Bloemenkamp DG, Van Den Bosch MA, Mali WP et al (2002) Novel risk factors for peripheral arterial disease in young women. Am J Med 113:462-467

13. Bostanci N, Belibasakis GN (2012) Porphyromonas gingivalis: an invasive and evasive opportunistic oral pathogen. FEMS Microbiol Lett 333:1-9

14. Calapkorur MU, Alkan B, Tasdemir Z et al (2017) Association of peripheral arterial disease with periodontal disease: analysis of inflammatory cytokines and an acute phase protein in gingival crevicular fluid and serum. J Periodont Res 52:532-539

15. Cavrini F, Sambri V, Moter A et al (2005) Molecular detection of treponema denticola and porphyromonas gingivalis in carotid and aortic atheromatous plaques by FISH: report of two cases. JMed Microbiol 54:93-96

16. Cetin C, Baumgartner I (2004) Die periphere arterielle Verschlusskrankheit (PAVK). In: Swiss Medical Forum. EMH Media, S216-223

17. Chen Y-W, Umeda M, Nagasawa T et al (2008) Periodontitis may increase the risk of peripheral arterial disease. Eur J Vasc Endovasc Surg 35:153-158

18. Cho D-H, Song I-S, Choi J et al (2020) Risk of peripheral arterial disease in patients with periodontitis: a nationwide, population-based, matched cohortstudy. Atherosclerosis 297:96-101

19. Cury EZ, Santos VR, Maciel SDS et al (2018) Lipid parameters in obese and normal weight patients with or without chronic periodontitis. Clin Oral Invest 22:161-167

20. Diehm C, Schuster A, Allenberg JR et al (2004) High prevalence of peripheral arterial disease and comorbidity in 6880 primary care patients: crosssectional study. Atherosclerosis 172:95-105 
21. Duran-Pinedo AE, Chen T, Teles R et al (2014) Community-wide transcriptome of the oral microbiome in subjects with and without periodontitis. ISME J 8:1659

22. Ford PJ, Gemmell E, Hamlet SM et al (2005) Cross-reactivity of GroEL antibodies with human heat shock protein 60 and quantification of pathogens in atherosclerosis. Oral Microbiol Immunol 20:296-302

23. Gerhard-Herman MD, Gornik HL, Barrett $C$ et al (2017) 2016 AHA/ACC guideline on the management of patients with lower extremity peripheral artery disease: a report of the American college of cardiology/American heart association task force on clinical practice guidelines. J Am Coll Cardiol69:e71-e126

24. Hamburg NM, Creager MA (2017) Pathophysiology of intermittent claudication in peripheral artery disease. Circ J 81(3):281-289

25. Hung H-C, Willett W, Merchant A et al (2003) Oral health and peripheral arterial disease. Circulation 107:1152-1157

26. Jeffcoat MK, Geurs NC, Reddy MS et al (2001) Periodontal infection and preterm birth: results of a prospective study. J Am Dent Assoc 132:875-880

27. Jordan AR, Micheelis W (2016) Fünfte Deutsche Mundgesundheitsstudie (DMS V). In: Materialienreihe, Bd. 35. Deutscher Ärzteverlag, Köln, S617

28. Kaschwich M, Behrendt CA, Heydecke G, Bayer A, Debus ES, Seedorf U, Aarabi G (2019) The association of periodontitis and peripheral arterial occlusive disease - a systematic review. Int J Mol Sci 20:2936. https://doi.org/10.3390/ ijms20122936

29. Kassebaum NJ, Bernabe E, Dahiya $M$ et al (2014) Global burden of severe periodontitis in 1990-2010: a systematic review and metaregression. J Dent Res 93:1045-1053

30. Kebschull A, Demmer R, Papapanou P (2010) "Gum bug, leave my heart alone!"-epidemiologic and mechanistic evidence linking periodontal infections and atherosclerosis. J Dent Res 89:879-902

31. KohalR, Lutter G, Dennison Detal (2001) Marginale Parodontitis und kardiovaskulare Erkrankungen. Schweiz Monatsschr Zahnmed 111:445-450

32. Kozarov E, Sweier D, Shelburne C et al (2006) Detection of bacterial DNA in atheromatous plaques by quantitative PCR. Microbes Infect 8:687-693

33. Kreutzburg T, Peters F, Riess HC et al (2020) Editor's choice-comorbidity patterns among patients with peripheral arterial occlusive disease in Germany: a trend analysis of health insurance claims data. Eur J Vasc Endovasc Surg 59:59-66

34. Lawall H, Diehm C, Hoffmann U et al (2015) Periphere arterielle Verschlusskrankheit: Epidemiologie, Komorbidität und Prognose. Dtsch Med Wochenschr 140:1798-1802

35. Leishman SJ, Ford PJ, Do HL et al (2012) Periodontal pathogen loadand increased antibody response to heat shock protein 60 in patients with cardiovascular disease. J Clin Periodontol 39:923-930

36. Leite FR, Nascimento GG, Scheutz F et al (2018) Effect of smoking on periodontitis: a systematic review and meta-regression. Am J Prev Med 54:831-841

37. Lu B, Parker D, Eaton CB (2008) Relationship of periodontal attachment loss to peripheral vascular disease: an analysis of NHANES 1999-2002 data. Atherosclerosis 200:199-205

38. Mcdermott MM (2015) Lower extremity manifestations of peripheral artery disease: the pathophysiologic and functional implications of leg ischemia. Circ Res 116:1540-1550

39. Mendez MV, Scott T, Lamorte W et al (1998) An association between periodontal disease and peripheral vascular disease. Am J Surg 176:153-157

40. Miao CY, Li ZY (2012) The role of perivascular adipose tissue in vascular smooth muscle cell growth. Br JPharmacol 165:643-658

41. Molloy J, Wolff LF, Lopez-Guzman A et al (2004) The association of periodontal disease parameters with systemic medical conditions and tobacco use JClin Periodontol 31:625-632

42. Müller-Wille R, Wohlgemuth W (2017) Lebensqualität nach endovaskulärer Intervention bei PAVK. Gefässchirurgie 22:165-168

43. Muñoz-Torres FJ, Mukamal KJ, Pai JK et al (2017) Relationship between tooth loss and peripheral arterial disease among women. J Clin Periodontol 44:989-995

44. Okuda K, Ishihara K, Nakagawa T et al (2001) Detection of Treponema denticola in atherosclerotic lesions. JClin Microbiol 39:1114-1117

45. Ott SJ, El Mokhtari NE, Musfeldt M et al (2006) Detection of diverse bacterial signatures in atherosclerotic lesions of patients with coronary heart disease. Circulation 113:929-937

46. Rossol M, Heine H, Meusch U et al (2011) LPSinduced cytokine production in human monocytes and macrophages. Crit Rev Immunol 31:379-446

47. Shoenfeld Y, Sherer Y, Harats D (2001) Artherosclerosis as an infectious, inflammatory and autoimmune disease. Trends Immunol 22:293-295

48. Slade G, Offenbacher S, Beck J et al (2000) Acutephase inflammatory response to periodontal disease in the US population. JDent Res 79:49-57

49. Song P, Rudan D, Zhu Y et al (2019) Global, regional, and national prevalence and risk factors for peripheral artery disease in 2015: an updated systematic review and analysis. Lancet Glob Health 7:e1020-e1030

50. Soto-Barreras U, Olvera-Rubio JO, Loyola-Rodriguez JP et al (2013) Peripheral arterial disease associated with caries and periodontal disease. JPeriodontol 84:486-494

51. Van Dyke TE (2017) Pro-resolving mediators in the regulation of periodontal disease. Mol Aspects Med 58:21-36

52. Wang J, Geng X, Sun J et al (2019) The risk of periodontitis for peripheral vascular disease: a systematic review. Rev Cardiovasc Med 20:81-89

53. Ward RP, Goonewardena SN, Lammertin G et al (2007) Comparison of the frequency of abnormal cardiac findings by echocardiography in patients with and without peripheral arterial disease. Am J Cardiol 99:499-503

54. Wohlgemuth W, Niechzial M, Nagel E et al (2003) Die Messung der gesundheitsbezogenen Lebensqualität bei der peripheren arteriellen Verschlusskrankheit. In: RöFo-Fortschritte auf dem Gebiet der Röntgenstrahlen und der bildgebenden Verfahren. Thieme, Stuttgart, New York, S169-175

55. Yang S, Zhao LS, Cai C et al (2018) Association between periodontitis and peripheral artery disease: a systematic review and meta-analysis. BMC Cardiovasc Disord 18:141
Akademikerkinder stehen stärker unter Stress

Der Studienbeginn ist eine aufregende Phase. Kinder aus Akademikerhaushalten haben während dieser Zeit allerdings deutlich mehr Stress als solche aus NichtAkademikerfamilien. Das hat das schweizerisch-deutsche Forschungsteam, Prof. Dr. Alex Bertrams von der Universität Bern und Dr. Nina Minkley von der Ruhr-Universität Bochum (RUB), durch Haaranalysen unter Studienanfängerinnen herausgefunden.

In Stresssituationen schüttet der Körper vermehrt das Hormon Cortisol aus, das auch in die wachsenden Haare gelangt und sich dort - im Falle von über längere Zeit hohen Werten - einlagert. Untersucht man die Haare, kann man daran erkennen, in welchen Phasen eine Person vermehrt Stress hatte.

Um herauszufinden, ob sich das Stresslevel bei jungen Menschen mit verschiedenen familiären Hintergründen zu Beginn eines Hochschulstudiums unterscheidet, gewann das Forschunsgsteam insgesamt 71 Testpersonen. „Die einzigen Einschlusskriterien waren, dass sie im ersten Semester sein mussten, und dass sie ausreichend langes Haar hatten", erklärt Dr. Nina Minkley.

Es stellte sich heraus, dass Studienanfängerinnen, in denen mindestens ein Elternteil selbst über einen Hochschulabschluss verfügte, höhere Stresslevel aufwiesen als solche aus Nicht-Akademikerhaushalten, obwohl sie sich in anderen Punkten nicht unterschieden. Das Forschungsteam interpretiert dieses Ergebnis als Zeichen dafür, dass Akademikerkinder stärker unter Druck sind weil ein Versagen im Studium einen Statusverlust für sie und ihre Familie bedeuten würde.

Originalpublikation: Bertrams, A. \& Minkley, N.: Preliminary indications that first semester students from academic households exhibit higher hair cortisol concentrations than their peers from non-academic households, in: Frontiers in Psychiatry 2020, DOI: 10.3389/fpsyt.2020.00580

Quelle: Ruhr-Universität Bochum 